\title{
An imaging agent to detect androgen receptor and its active splice variants in prostate cancer
}

Yusuke Imamura, ${ }^{1}$ Amy H. Tien, ${ }^{1}$ Jinhe Pan, ${ }^{2}$ Jacky K. Leung, ${ }^{1}$ Carmen A. Banuelos, ${ }^{1}$ Kunzhong Jian, ${ }^{3}$ Jun Wang, ${ }^{1}$ Nasrin R. Mawji, Javier Garcia Fernandez, ${ }^{3}$ Kuo-Shyan Lin, ${ }^{2}$ Raymond J. Andersen, ${ }^{3}$ and Marianne D. Sadar'

${ }^{1}$ Cenome Sciences Centre and ${ }^{2}$ Department of Molecular Oncology, British Columbia Cancer Agency, Vancouver, Canada. ${ }^{3}$ Chemistry and Earth, Ocean, and Atmospheric Sciences, University of British Columbia, Vancouver, Canada.

Constitutively active splice variants of androgen receptor (AR-Vs) lacking ligand-binding domain (LBD) are a mechanism of resistance to androgen receptor LBD-targeted (AR LBD-targeted) therapies for metastatic castration-resistant prostate cancer (CRPC). There is a strong unmet clinical need to identify prostate cancer patients with AR-V-positive lesions to determine whether they will benefit from further AR LBD-targeting therapies or should receive taxanes or investigational drugs like EPI-506 or galeterone. Both EPI-506 (NCT02606123) and galeterone (NCT02438007) are in clinical trials and are proposed to have efficacy against lesions that are positive for AR-Vs. AR activation function-1 (AF-1) is common to the $\mathrm{N}$-terminal domains of fulllength $A R$ and $A R-V s$. Here, we provide proof of concept for developing imaging compounds that directly bind AR AF-1 to detect both AR-Vs and full-length AR. ${ }^{123}$ I-EPI- 002 had specific binding to AR AF-1, which enabled direct visualization of CRPC xenografts that express full-length AR and AR-Vs. Our findings highlight the potential of ${ }^{123} \mathrm{I}-\mathrm{EPI}-002$ as an imaging agent for the detection of full-length AR and AR-Vs in CRPC.

Conflict of interest: Y. Imamura, A.H. Tien, C.A. Banuelos, J. Wang, N.R. Mawji, K. Jian, J.G. Fernandez, R.J. Andersen, and M.D. Sadar are inventors and have licensed the technology to ESSA Pharma Inc. C.A. Banuelos, J. Wang, N.R. Mawji, J.G. Fernandez, R.J. Andersen, and M.D. Sadar all have shares in ESSA Pharma Inc., and R.J. Andersen and M.D. Sadar are directors and officers and receive consulting fees.

Submitted: March 31, 2016

Accepted: June 9, 2016

Published: July 21, 2016

Reference information:

JCI Insight. 2016;1(11):e87850.

doi:10.1172/jci.insight.87850.

\section{Introduction}

Most castration-resistant prostate cancer (CRPC) continues to be dependent on transcriptionally active androgen receptor (AR). Recently, powerful agents in the arsenal of hormone therapies for advanced prostate cancer, such as abiraterone and enzalutamide, have been approved based upon 5- to 6-month improvements in overall survival. Approximately $20 \%-40 \%$ of patients with metastatic CRPC have primary resistance to enzalutamide or abiraterone (1-4), and patients that were originally responsive will all eventually fail. One mechanism of resistance to current hormone therapies is the presence of constitutively active splice variants of AR (AR-Vs) (5-7).

Full-length $\mathrm{AR}$ is a ligand-activated transcription factor with distinct functional domains that include the C-terminal ligand-binding domain (LBD), to which androgens and antiandrogens bind; the hinge region, which contains a nuclear translocation sequence; the DNA-binding domain (DBD), which binds to androgen response elements (AREs) in the enhancers/promoters of target genes; and the N-terminal domain (NTD), which contains activation function-1 (AF-1) that is essential for AR transcriptional activity $(8-10)$.

All current hormone therapies target the AR LBD either directly with antiandrogens or indirectly by reducing levels of androgen that bind to AR LBD. Constitutively active AR-Vs, such as V7 and V567es, lack a full LBD and are nonresponsive to both androgen and therapies targeting $\operatorname{AR~} \operatorname{LBD}(11,12)$. Abiraterone and enzalutamide increase expression of V7 in prostate cancer cells and xenografts $(7,12,13)$. AR-Vs mediate a growth advantage for prostate cancer in androgen-deprived conditions $(11,14)$ and are detected in CRPC tissues $(11,14,15)$. Of the more than 20 AR-Vs discovered $(16,17)$, V7 and V567es are clinically relevant, with levels of expression correlated to poor survival and CRPC (16). Detection of V7 in circulating tumor cells (CTCs) of prostate cancer patients is prognostic of resistance to enzalutamide and abiraterone (5), whereas V7-positive patients respond to taxanes (18). Thus, the ability to determine AR-Vs status of CRPC patients is vital to their clinical management to prevent patients from receiving futile, high-cost treatments and therapies (e.g., enzalutamide, $\$ 7,450 /$ month and abiraterone $\$ 5,000 /$ month). 


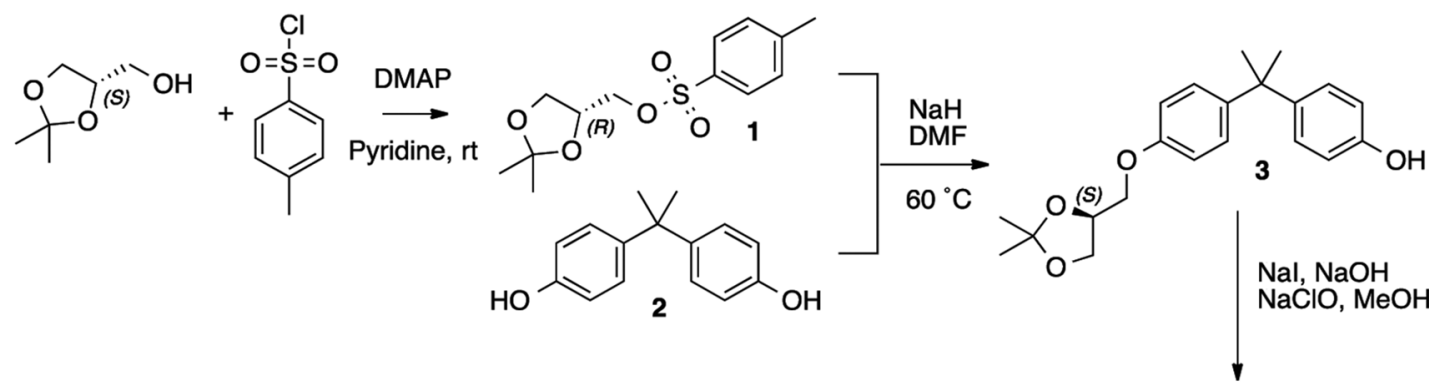

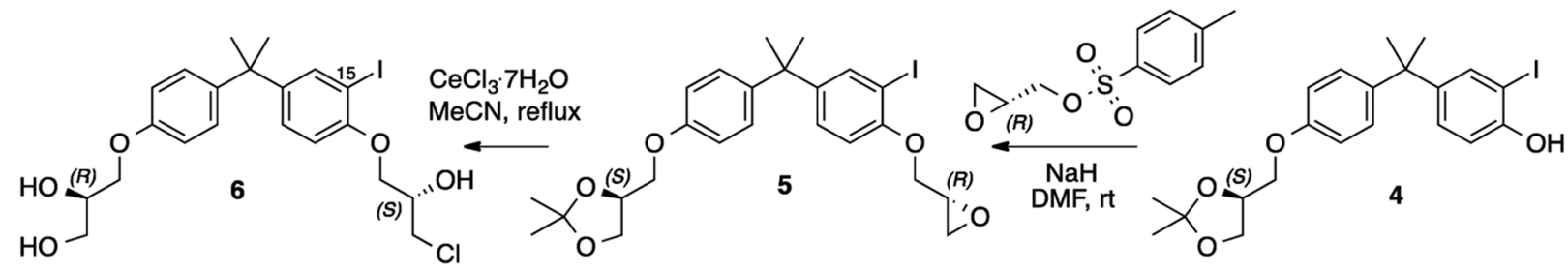

Figure 1. Five-step synthetic route to cold 15-iodoEPI-002 (I-EPI-002) starting from (S)-glycerol acetonide and bisphenol A [2].

Unfortunately, approaches to determine if a patient harbors metastases that express AR-Vs are not developed or validated, with each approach having significant shortcomings. Measurement of AR-Vs in CTCs is dependent on abilities to isolate/detect CTCs and the sensitivity of the assay to measure levels of AR-Vs. Current assays for AR-Vs in CTCs have limited reproducibility, sensitivity, and specificity, with the predictive value of the assay limited to only patients with detectable CTCs. Detection of CTCs is dependent on tumor volume and the presence of a surface antigen, such as HER2 (human epidermal growth factor receptor 2), EpCAM (epithelial cell adhesion molecule), or CD45; detection may not capture the entire CTC population. Circulating tumor-derived cell-free DNA (ctDNA) is also undergoing evaluation alongside CTCs for its utility in clinical practice $(19,20)$. Conceptually, ctDNA and/or ctRNA may be derived from primary tumors, metastatic lesions, CTCs, or from the plethora of benign tissues that are known to express AR-Vs (21). ctDNA analyses are restricted to measurable changes at the DNA level, and to date the clinical relevance of genomic rearrangement to generation of AR-Vs is lacking. Thus, there is an urgent need to develop clinical methods to determine expression of AR-Vs in metastatic CRPC. One possible approach is application of molecular imaging agents to detect AR-Vs. EPI small molecules provide an opportunity to develop such a prognostic imaging tool because they bind to AF-1 (22-24) that is common to both full-length AR and AR-Vs. EPI-001 and its stereoisomers (e.g., EPI-002) and analogs (e.g., EPI-506) were developed as AF-1 inhibitors to block transactivation of both full-length AR and AR-Vs. EPI-506 received investigational new drug approval from the FDA and Health Canada (i.e., acceptable safety profile at therapeutic doses) and is currently in clinical trials in the US and Canada for CRPC patients that have failed abiraterone and/or enzalutamide.

Here, we provide proof of concept for developing an analog of EPI for imaging full-length AR and AR-Vs in CRPC patients. We provide evidence that single-photon emission computed tomography-computed tomography (SPECT/CT) successfully and specifically imaged prostate cancer xenografts that express AR-Vs after tail vein administration of microdoses of radioiodine 123-EPI-002 ( ${ }^{123}$ I-EPI-002). To specifically reveal AR-Vs, a discordant distribution and/or discordant level of uptake between $16 \beta-\left[{ }^{18} \mathrm{~F}\right]$-fluoro-5 $\alpha$ dihydrotestosterone $\left({ }^{18} \mathrm{~F}\right.$-FDHT), which binds only full-length AR, and an imaging agent, such as ${ }^{123} \mathrm{I}-\mathrm{EPI}-002$, which binds AF-1 that is common to both full-length AR and AR-Vs, would indicate the expression of solely AR-Vs. Currently, this may be technically challenging. However, if such an imaging approach were developed, it could reveal patients with lesions that are positive for AR-Vs who may not benefit from further therapies that target AR LBD. Our findings suggest that an imaging probe targeted to AR AF-1 may aid in monitoring treatment responses to significantly affect the clinical management of the disease as well as provide insight into the role of all AR species in resistance mechanisms. 

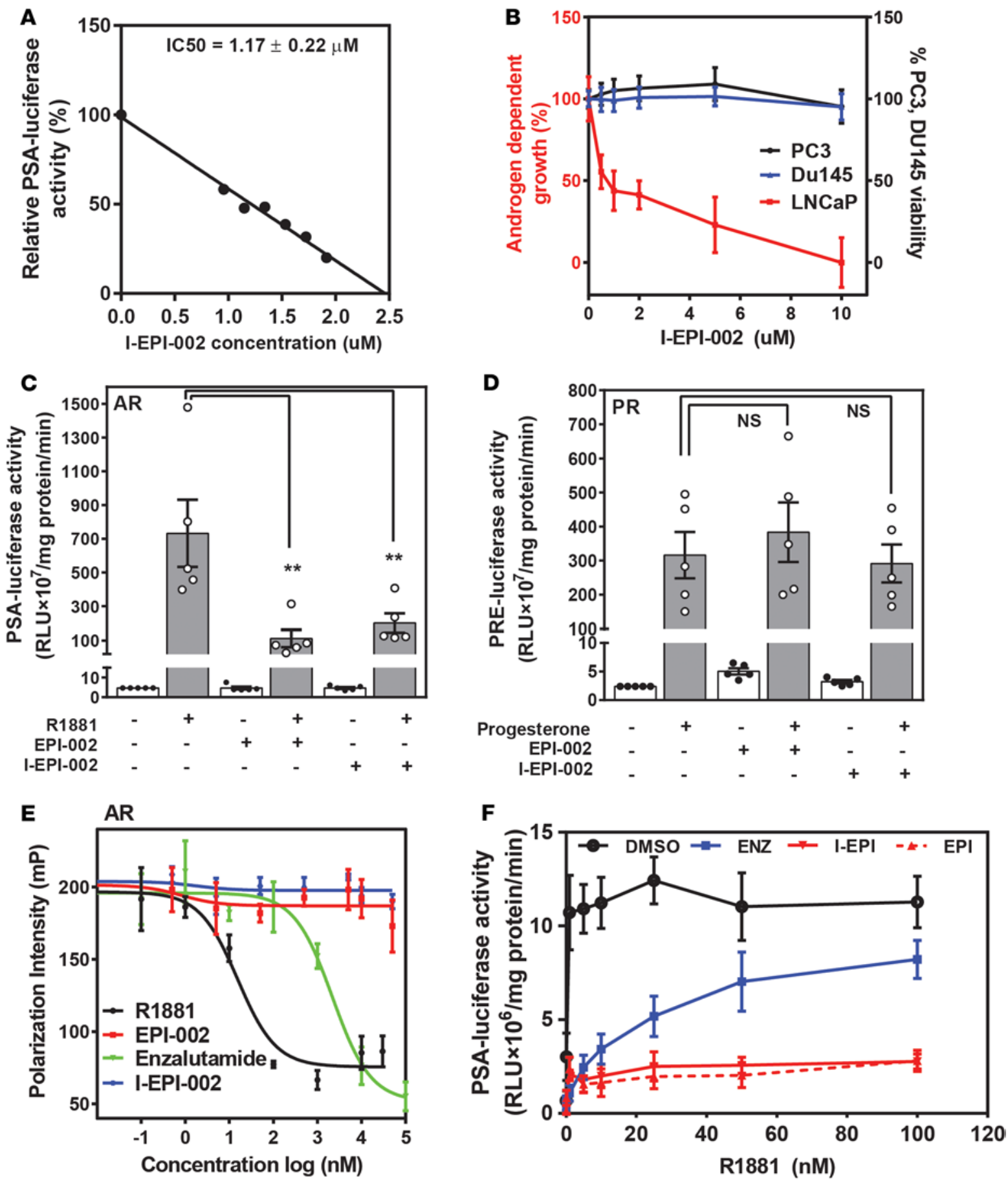

Figure 2. I-EPI-002 has better potency than EPI-002 for AR and maintains specificity. (A) Dose-dependent inhibition of androgen induced transcriptional activity of endogenous full-length AR in LNCaP cells by I-EPI-002. $n \geq 3$. Representative data are shown. (B) Effects of I-EPI-002 on androgen-dependent proliferation of LNCaP cells treated with R1881 compared with PC3 and DU145 cell viability. $n \geq 3$. Data represent mean \pm SD. (C) Effect of I-EPI-002 $(2 \mu \mathrm{M})$ vs. EPI-002 $(25 \mu \mathrm{M})$ on AR transcriptional activity in LNCaP cells that were transiently transfected with PSA(6.1 kb) luciferase reporter and induced with R1881 $(1 \mathrm{nM}) . n=5$. Data represent mean \pm SEM. (D) Effect of I-EPI-002 $(2 \mu \mathrm{M})$ vs. EPI-002 (25 $\mu$ M) on 4-pregnene-3,20-dione (Progesterone, $10 \mathrm{nM}$ ) induced PR transcriptional activity in LNCaP cells that were transiently transfected with PRE luciferase reporter and expression vector for PR $\beta . n=5$. Data represent mean \pm SEM. (E) A representative competition binding curve showing displacement of $1 \mathrm{nM}$ fluorescently labeled ligand from recombinant AR LBD $(25 \mathrm{nM})$ by enzalutamide and R1881 but not with I-EPI-002 or EPI-002. The experiment was repeated 3 times. (F) Androgen-induced AR-transcriptional activity measured in LNCaP cells transfected with PSA(6.1 kb) luciferase reporter and treated with vehicle (DMSO), enzalutamide (ENZ, $5 \mu \mathrm{M})$, I-EPI-002 $(5 \mu \mathrm{M})$, or EPI-002 $(25 \mu \mathrm{M})$ and increasing concentrations of R1881. $n=4$. Values shown are the mean \pm SD. I-EPI, I-EPI-002; EPI, EPI-002; ENZ, enzalutamide. (A-F) Each independent experiment was performed in triplicate. ${ }^{* *} P<0.01$. One-way ANOVA Dunnett's multiple comparison test was utilized. 


\section{Results}

I-EPI-002. EPI-001 and its analogs bind AF-1 (22) that is common to both full-length AR and AR-Vs. For an imaging agent to be successful, it must have excellent binding affinity for its target. The most potent stereoisomer of the EPI-001 mixture is EPI-002 (23), which was employed as the starting scaffold for synthesis of cold 15-IodoEPI002 (called I-EPI-002) (Figure 1 and described in the Supplemental Experimental Procedures; supplemental material available online with this article; doi:10.1172/jci.insight.87850DS1).

Iodination improves the potency of EPI-002 in functional assays. Iodination of EPI-002 (I-EPI-002) could potentially alter many of the properties of the parent compound EPI-002, such as potency, biological activity, and off-target effects. Since excellent binding affinity is critical for an imaging agent to be successful, we first tested whether iodination of EPI-002 improved or diminished its potency using well-established cell-based functional assays. Dose response curves using the AR-driven prostate-specific antigen (PSA) (6.1 $\mathrm{kb})$ luciferase reporter were used to calculate $\mathrm{IC}_{50}$ values for inhibition of endogenous $\mathrm{AR}$ transcriptional activity by I-EPI-002 compared with EPI-002 in LNCaP cells. I-EPI-002 had an $\mathrm{IC}_{50}$ of $1.17 \pm 0.22 \mu \mathrm{M}$ for inhibition of AR transcriptional activity (Figure 2A). This is comparable to that reported for the antiandrogen enzalutamide, which is considered to have strong affinity for the AR. At $1 \mu \mathrm{M}$, enzalutamide caused $67 \%$ inhibition of ARE( $4 \mathrm{x}$ ) luciferase activity (25). I-EPI-002 had an $\mathrm{IC}_{50}$ of approximately $1 \mu \mathrm{M}$ to block AR-driven proliferation of LNCaP cells in response to androgen (Figure 2B). I-EPI-002 had no effect on proliferation or viability of PC3 and DU145 human prostate cancer cells that do not express functional AR at up to 10 times the $\mathrm{IC}_{50}$ required to reduce AR-dependent proliferation, thereby supporting its specificity for AR. I-EPI-002 is 10 times more potent than EPI-002, which has $\mathrm{IC}_{50}$ in the range of $10 \mu \mathrm{M}$ for these cell-based assays $(22,23)$. Together, these data support that I-EPI-002 has improved potency compared with EPI-002 and are consistent with saturation transfer difference-NMR data, showing strong interaction of the bisphenyl rings of EPI with Tau-5 of AR AF-1 (24).

I-EPI-002 maintains specificity for AR. Imaging agents are administered at microdoses and thus must be highly specific for their targets. Therefore, we next examined whether I-EPI-002 would potentially interact with the proteins most highly related to AR in the human proteome, which are the other steroid receptors, progesterone receptor (PR), glucocorticoid receptor (GR), and estrogen receptor (ER). In addition to high sequence identity in the LBD and DBD of this class of receptors, they also interact with many of the same proteins, such as CBP (CREB-binding protein) and SRC1-3. A sensitive method to determine if I-EPI-002 interacts with any of these other receptors is to measure their transcriptional activities. Therefore, reporter gene assays were used to determine if I-EPI-002 would inhibit PR, GR, or ER transcriptional activities. Cells were cotransfected with expression plasmids for full-length human PR $\beta, G R, E R \alpha$, and their respective reporters and then treated with ethanol vehicle, 4-pregnene-3,20 dione (progesterone) (PR $\beta$ ), dexamethasone (GR), or estradiol (E2) (ER $\alpha$ ). At a concentration of $\sim 2 \mu \mathrm{M}$, I-EPI-002 inhibited AR transcriptional activity to similar levels (75\%) to that achieved with $25 \mu \mathrm{M}$ EPI-002 (Figure 2C). Importantly, I-EPI-002 did not inhibit the transcriptional activities of PR, GR, or ER in response to their cognate ligands (Figure 2D and Supplemental Figure 1, A and B). These data provide evidence that I-EPI-002 does not have general effects on transcription or translation because it did not inhibit induction of PRE (progesterone response element), GRE (glucocorticoid response element), or ERE (estrogen response element) luciferase reporters in response to ligand. Thus, I-EPI-002 appears to maintain specificity for AR.

I-EPI-002 does not interfere with ligand binding. EPI analogs bind specifically to AR AF-1 without being affected by, or interfering with, ligand binding to $\operatorname{AR} \operatorname{LBD}(22,23)$. This is an important attribute for an imaging agent for CRPC, because AR LBD may be occupied by antiandrogen or steroid/androgen. To test whether I-EPI-002 maintained this property and does not affect ligand binding to AR LBD, we employed the fluorescence polarization assay. This assay measures competition of a test compound for binding of fluoromone to recombinant AR LBD or other related steroid hormone receptor LBDs. R1881 and the antiandrogen enzalutamide both bound to the AR LBD to compete with the fluoromone as expected (Figure 2E). I-EPI-002 behaved consistently to EPI-002 and did not prevent ligand-binding to the LBDs of AR or any steroid hormone receptors tested (Supplemental Figure 1, C-F). Consistent with these data, I-EPI-002 and EPI-002 maintained effective inhibition of AR transcriptional activity with increasing concentrations of androgen (Figure 2F), unlike enzalutamide, which markedly lost potency, as expected for a competitive antiandrogen. These data support that imaging with a molecular probe targeted to AR AF-1 should not be affected by an occupied AR LBD.

I-EPI-002 targets AR-Vs. Constitutively active AR-Vs that lack LBD are detected in clinical samples of CRPC, and levels of expression are correlated with poor prognosis and resistance to abiraterone 


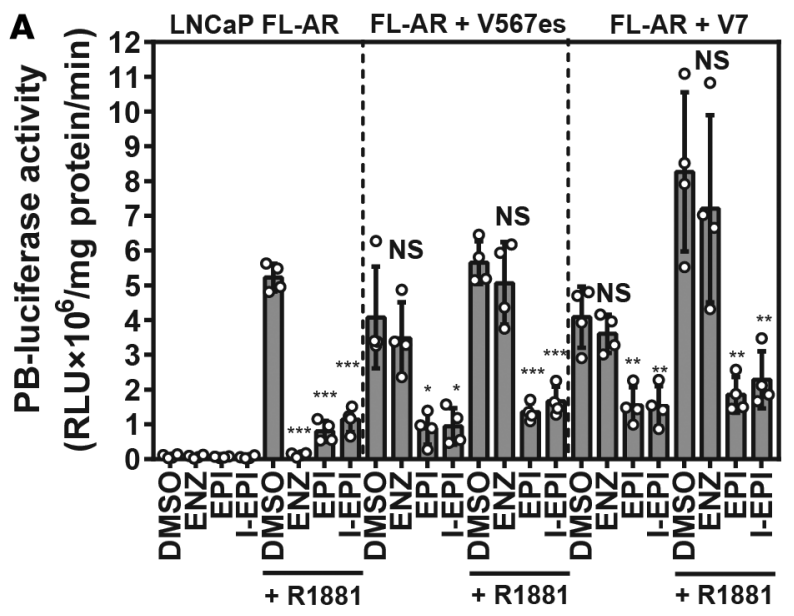

B

C
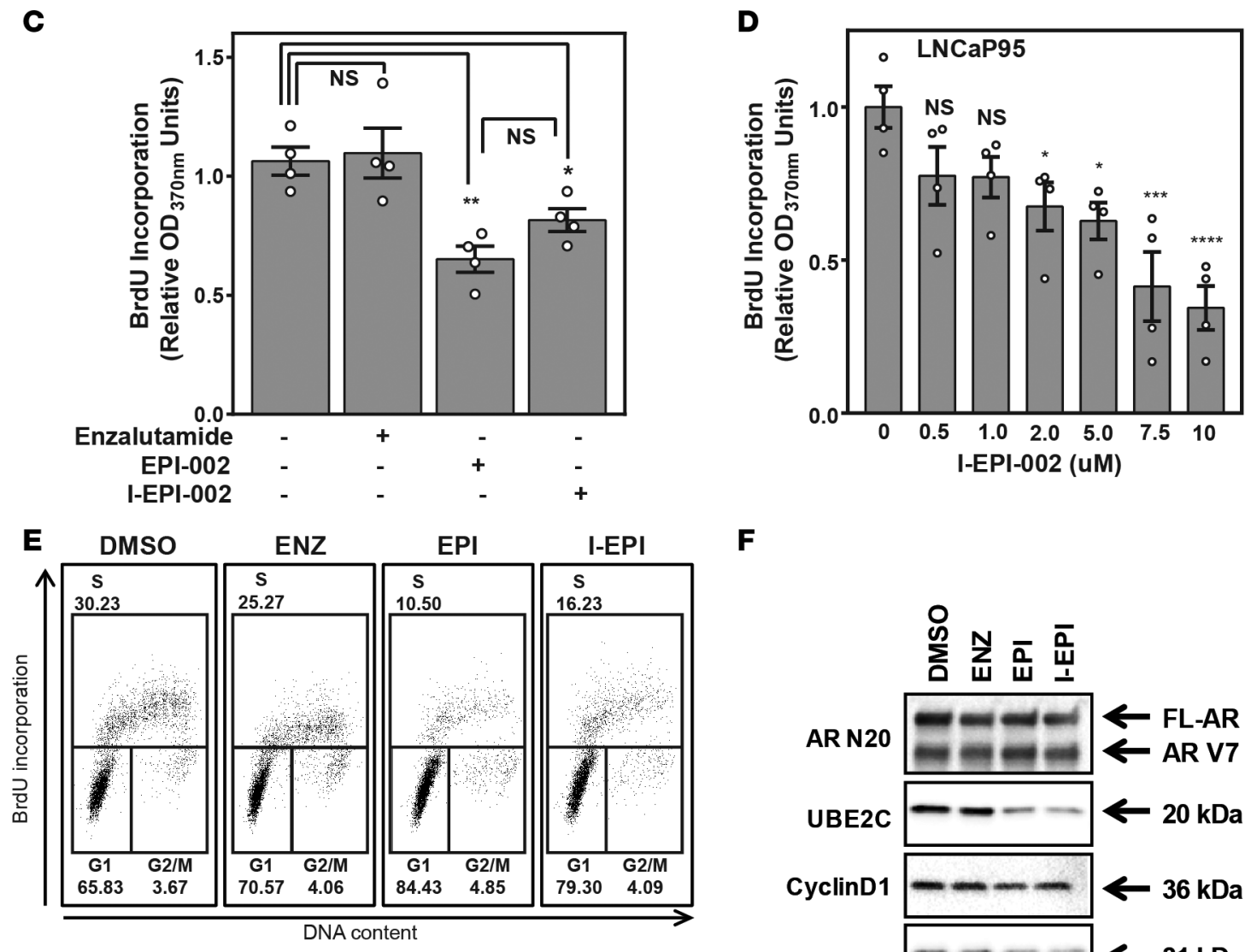

$\mathbf{F}$
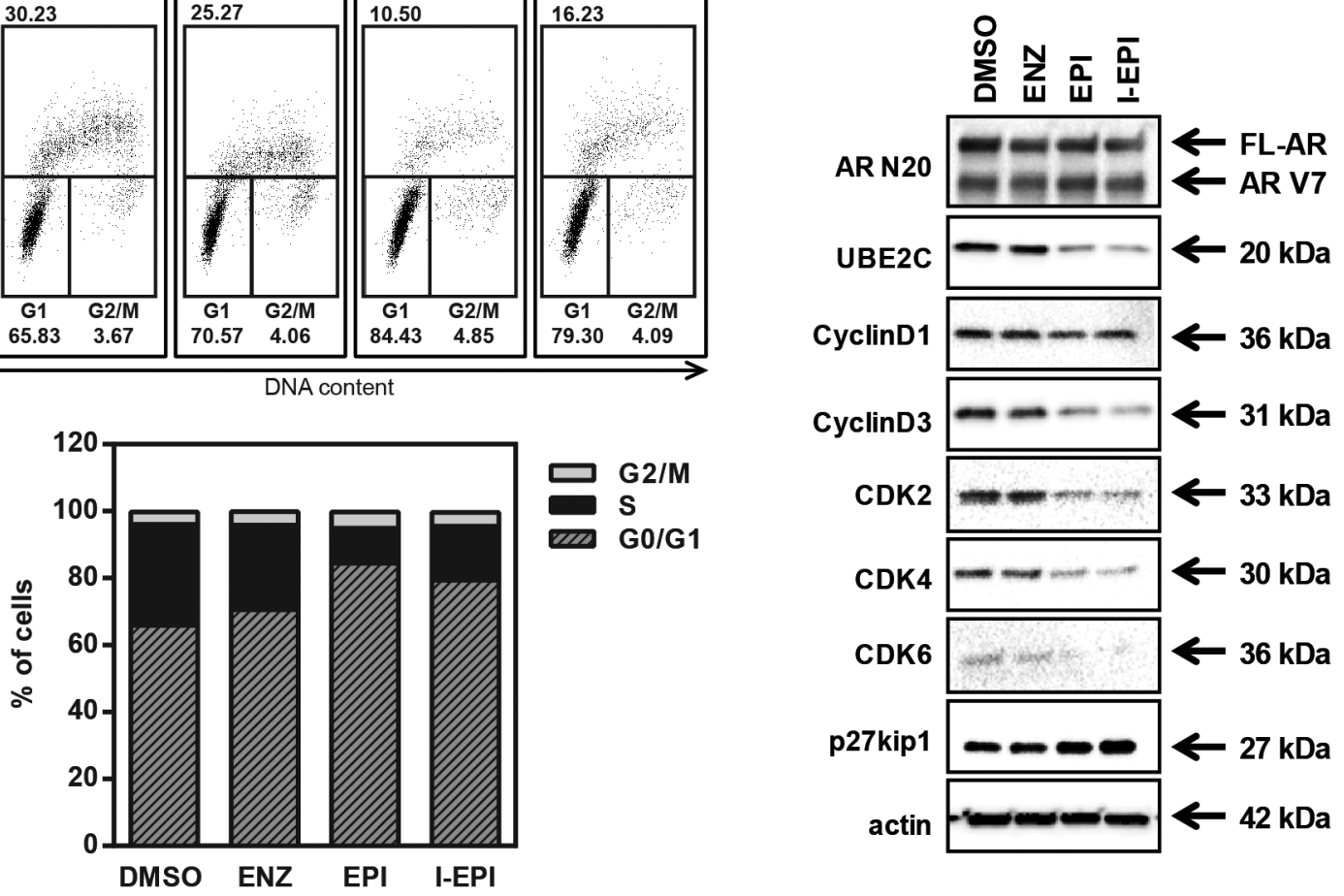
Figure 3. I-EPI-002 inhibits full-length AR and constitutively active AR-Vs. (A) Probasin luciferase activity in LNCaP cells with endogenous full-length AR (left), with both full-length AR and AR-V567es (middle), or with both full-length AR and V7 (right). Cells were treated with $2 \mu \mathrm{M} \mathrm{I-EPI-002,} 25 \mu \mathrm{M}$ EPI002 , or $5 \mu \mathrm{M}$ enzalutamide for 1 hour prior to treatment with or without $1 \mathrm{nM}$ R1881 for 24 hours. Data represent mean \pm SD from 4 independent experiments, each performed in triplicate. (B) A representative blot showing protein levels of full-length AR, V567es, and V7 from samples in $\mathbf{A}$ detected using AR-N20 antibody. (C) LNCaP95 cells treated with enzalutamide $(5 \mu \mathrm{M})$, EPI-002 $(25 \mu \mathrm{M})$, or I-EPI-002 $(2 \mu \mathrm{M})$ for 2 days. $n=4$. Data represent mean \pm SEM. Proliferation was assessed by BrdU incorporation. (D) LNCaP95 cells treated with different concentrations of I-EPI-002 for 2 days. $n=4$. Data represent mean \pm SEM. (E) Inhibition of DNA synthesis in LNCaP95 cells by I-EPI-002 $(5 \mu \mathrm{M})$, EPI-002 (25 $\mu \mathrm{M})$, or enzalutamide $(5 \mu \mathrm{M})$ treatment for 48 hours. Bivariate plots show cell cycle distribution of a representative experiment. The stacked graph (below) represents the average results from 3 independent experiments. The percentage of cells in each phase of cell cycle was calculated. (F) Effects of enzalutamide, EPI-002, and I-EPI-002 on cell cycle-regulated proteins in LNCaP95 cells are shown by Western blot analyses. LNCaP95 cells were serum starved for 24 hours and then treated with DMSO, enzalutamide $(5 \mu \mathrm{M})$, EPI-002 $(25 \mu \mathrm{M})$, or I-EPI-002 $(5 \mu \mathrm{M})$ for 48 hours (refer to full and uncut gel images in Supplemental Materials). I-EPI, I-EPI-002; EPI, EPI-002; ENZ, enzalutamide. ${ }^{*} P<0.05,{ }^{* *} P<0.01,{ }^{* * *} P<0.001,{ }^{* * *} P<0.0001$. One-way ANOVA Dunnett's multiple comparison test was utilized.

and enzalutamide, which target the $\operatorname{AR} \operatorname{LBD}(5,11,14,16,26,27)$. To ensure that the addition of the iodo to EPI-002 had no detrimental effect on its ability to interact with truncated AR-Vs, we performed direct comparison of I-EPI-002, EPI-002, and enzalutamide on solely endogenous full-length AR or endogenous full-length AR combined with V567es or V7. In the absence of V567es or V7, enzalutamide inhibited full-length AR induced by androgen, as measured with AR-driven probasin luciferase reporter (Figure 3A, left). Consistent with previous studies, enzalutamide had no effect in blocking AR transcriptional activity, either in the presence or absence of androgen, when V567es or V7 was expressed (Figure 3A, middle and right). I-EPI-002 showed good inhibition against full-length AR as well as mixed populations of full-length AR with variant V567es or V7 at concentrations 10 times lower than those of EPI-002. This suggests that I-EPI-002 interacts with the truncated AR variants, consistent with EPI-002, but with better potency. Western blot analysis using an antibody against the AR NTD confirmed the approximate 1:1 ratio of endogenous full-length AR to ectopic V567es or V7 in whole cell lysates of cells treated with I-EPI-002, EPI-002, and enzalutamide (Figure 3B). I-EPI-002 was next tested to examine if it had effects on AR-V-driven proliferation. LNCaP95 cells are enzalutamide resistant $(12,28)$ and increase proliferation in the absence of androgen by a mechanism driven by AR-Vs. I-EPI-002 $(2 \mu \mathrm{M})$ decreased proliferation of LNCaP95 cells as well as EPI-002 $(25 \mu \mathrm{M})$, whereas enzalutamide $(5 \mu \mathrm{M})$ had no effect (Figure 3C). I-EPI-002 had an $\mathrm{IC}_{50}$ of $6.89 \pm 2.85 \mu \mathrm{M}$ for inhibition of LNCaP95 cell proliferation (Figure 3D). Consistent with these data, cell cycle analysis revealed that EPI analogs caused $\mathrm{G}_{0} /$ $\mathrm{G}_{1}$ arrest. Approximately $25 \%-30 \%$ of cells treated with enzalutamide or DMSO vehicle were in S phase (Figure 3E). Whereas I-EPI-002 or EPI-002 decreased S phase cells by approximately 2-fold or more with a concomitant increase of cells in $\mathrm{G}_{1}$ phase. Western blot analysis of cell cycle $\mathrm{G}_{1} / \mathrm{S}$-related proteins revealed that EPI analogs decreased the expression of UBE2C (ubiquitin-conjugating enzyme E2C), an AR-V7-regulated protein; cyclinD3; and cyclin-dependent kinases (CDKs). EPI analogs increased protein expression of the CDK inhibitor p27kip1. Importantly, enzalutamide had no effect on the levels of expression of these proteins, which was consistent with these cells being resistant to enzalutamide (Figure 3F). Together, these data support that I-EPI-002 targets full-length AR and AR-Vs.

Synthesis of ${ }^{123} \mathrm{I}$-EPI-002 imaging probe. The preparation of $\left[{ }^{123} \mathrm{I}\right] 15$-iodoEPI002 is shown in Figure $4 \mathrm{~A}$. The radiosynthesis involves three reactions. First, the $\left.{ }^{123} \mathrm{I}\right]$ iodoacetonide 7 was prepared in $90 \% \pm 10 \%$ radiochemical yield via aromatic electrophilic iodination reaction using chloramines- $\mathrm{T}$ as the oxidizing reagent and the acetonide 3 as the precursor. The isolated $\left[{ }^{123} \mathrm{I}\right]$ iodoacetonide was subsequently coupled with (2R)-(-)-glycidyl tosylate to form $\left[{ }^{123} \mathrm{I}\right]$ iodoepoxide 8 in $37 \% \pm 17 \%$ radiochemical yield. At the final step, $\left[{ }^{123} \mathrm{I}\right] 15$-iodoEPI002 was obtained in $70 \% \pm 11 \%$ radiochemical yield by hydrolysis of the $\left[{ }^{123} \mathrm{I}\right]$ iodoepoxide 8 with $\mathrm{CeCl}_{3} \cdot 7 \mathrm{H}_{2} \mathrm{O}$. Due to multiple radiolabeling and HPLC purification steps, $\left.{ }^{[23} \mathrm{I}\right] 15$-iodoEPI002 ( ${ }^{123}$ I-EPI-002) was isolated in an overall $14 \% \pm 4 \%(n=12)$ decay-corrected yield in a 4.5 -hour synthesis time with $>99 \%$ radiochemical purity and $3.4 \pm 1.0 \mathrm{Ci} / \mu \mathrm{mol}$ specific activity at the end of synthesis.

${ }^{123}$ I-EPI-002 binds to AF-1 in the AR NTD in cells. EPI-002 and analogs bind to AR AF-1 $(22,23)$ and specifically to Tau-5 in the AF-1 region (24). Here, we show that ${ }^{123}$ I-EPI-002 binds to recombinant full-length AR (Figure 4B). To determine if ${ }^{123} \mathrm{I}$-EPI-002 binds the same site as EPI-002, AF-1 protein was preincubated with excess cold EPI-002 prior to addition of radioactive ${ }^{123}$ I-EPI-002 probe. Excess cold EPI-002 displaced binding of ${ }^{123} \mathrm{I}$-EPI-002 to AF-1, thereby suggesting they bind to the same site (Figure 4C). Ultimately to ensure specific binding of ${ }^{123}$ I-EPI-002 to endogenous AR in cells, LNCaP95 cells were treated with ${ }^{123}$ I-EPI-002 for 16 hours, followed by SDS-PAGE and Western blot analysis. ${ }^{123}$ I-EPI-002 binds covalently 
A
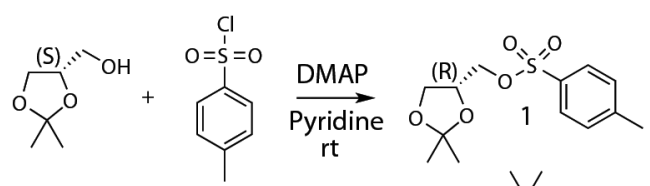<smiles>CC(C)(c1ccc(O)cc1)c1ccc(O)cc1</smiles>

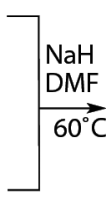<smiles>C[C@H]1[C@H](Oc2ccc(C(C)(C)c3ccc(O)cc3)cc2)COC1(C)C</smiles>

chloramine-T phosphate buffer

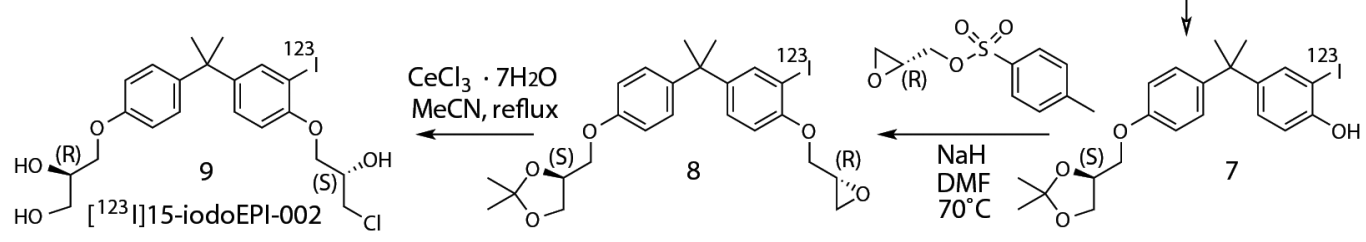

B

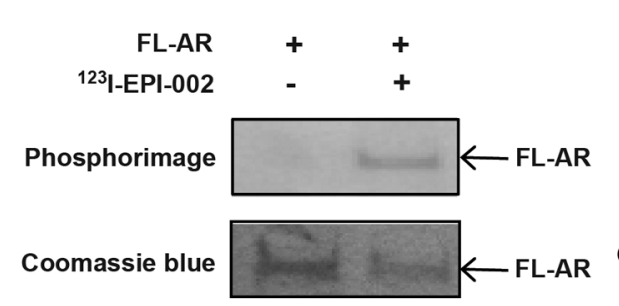

C

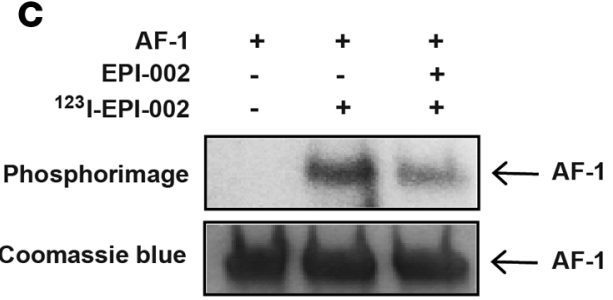

D

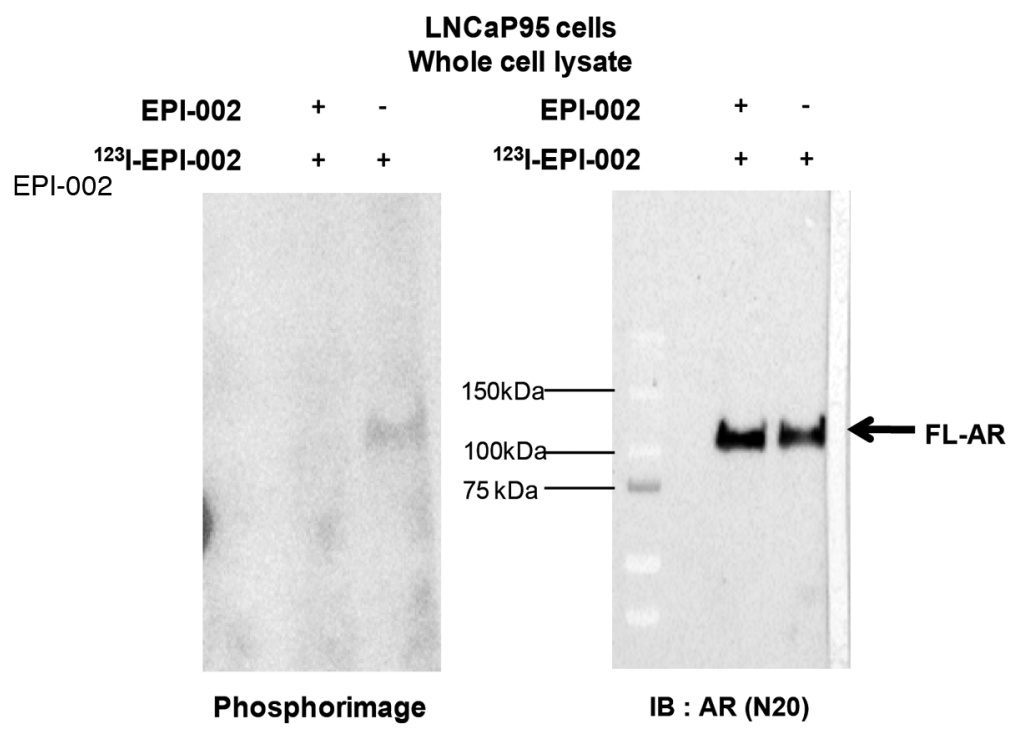

Figure 4. Synthesis of ${ }^{123}$ I-EPI-002 and its binding to AR AF-1. (A) Synthetic route to [23I]15-iodoEPI-002 starting from $(R)$-tosylglycerolacetonide[1] and bisphenol $\mathrm{A}[1]$ and using $\mathrm{Na}^{123}$ as a source of radiolabeled iodine. (B) Recombinant full-length AR protein was incubated with ${ }^{123}$ |-EPI-002 for 16 hours at room temperature. Binding of ${ }^{123}$ |-EPI-002 to AR was detected by SDS-PAGE followed by phosphorimaging. Coomassie blue staining was used to provide an indication of equal loading. (C) Recombinant AF-1 protein was incubated with vehicle (DMSO) or EPI-002 (60 $\mu \mathrm{M})$ for 6 hours at room temperature prior to addition of ${ }^{123}$ I-EPI-002 (200 $\left.\mu \mathrm{Ci}\right)$ and then incubated for 16 hours more prior to SDS-PACE and phosphorimaging. Less binding of ${ }^{123}$-EPI-002 was observed when AF-1 was preincubated with excess cold EPI-002. Coomassie blue staining was used to provide an indication of equal loading (refer to full and uncut gel images in Supplemental Materials). (D) Binding of ${ }^{123}$-EPI-002 to endogenous AR in LNCaP95 cells that were incubated with or without $25 \mu \mathrm{M}$ EPI- 002 overnight at $37^{\circ} \mathrm{C}$ prior to harvesting cells. Whole cell lysates were used for SDS-PAGE to reveal ${ }^{123}$-EPI-002 covalently bound to full-length AR in the LNCaP95 cells, as detected by phosphorimage. Western blot analysis detection of bands corresponding to AR for radiolabeled phosphorimaged bands. $n \geq 3$ for B, C, and $\mathbf{D}$ binding experiments. 
Table 1. Biodistribution of ${ }^{123}$ I-EPI-002 compound

\begin{tabular}{|c|c|c|c|c|c|c|}
\hline & $\mathrm{O} h(n=3)$ & $1 \mathrm{~h}(n=6)$ & $2 \mathrm{~h}(n=7)$ & $4 h(n=5)$ & $6 h(n=6)$ & $12 \mathrm{~h}(n=6)$ \\
\hline Positive tumor (LNCaP95) & $0.17 \pm 0.06$ & $2.19 \pm 0.45$ & $1.13 \pm 0.16$ & $1.04 \pm 0.14$ & $0.52 \pm 0.10$ & $0.15 \pm 0.08$ \\
\hline Negative tumor (PC3) & $0.19 \pm 0.06$ & $0.69 \pm 0.40$ & $0.35 \pm 0.14$ & $0.50 \pm 0.17$ & $0.49 \pm 0.01$ & $0.13 \pm 0.13$ \\
\hline Blood & $12.57 \pm 2.03$ & $0.74 \pm 0.30$ & $0.31 \pm 0.08$ & $0.25 \pm 0.07$ & $0.17 \pm 0.03$ & $0.07 \pm 0.04$ \\
\hline Kidney & $5.57 \pm 2.37$ & $3.07 \pm 0.90$ & $1.43 \pm 0.58$ & $1.05 \pm 0.11$ & $0.65 \pm 0.24$ & $0.16 \pm 0.08$ \\
\hline Spleen & $1.15 \pm 1.04$ & $0.96 \pm 0.23$ & $0.71 \pm 0.34$ & $0.55 \pm 0.24$ & $0.30 \pm 0.18$ & $0.07 \pm 0.03$ \\
\hline Small intestine & $2.44 \pm 1.05$ & $7.43 \pm 5.25$ & $6.29 \pm 3.72$ & $6.25 \pm 2.18$ & $2.37 \pm 1.43$ & $2.14 \pm 1.49$ \\
\hline Stomach & $1.80 \pm 1.60$ & $1.73 \pm 0.69$ & $0.68 \pm 0.31$ & $0.29 \pm 0.19$ & $0.19 \pm 0.08$ & $1.78 \pm 1.27$ \\
\hline Heart & $7.62 \pm 1.86$ & $2.25 \pm 0.50$ & $1.04 \pm 0.22$ & $0.88 \pm 0.18$ & $0.51 \pm 0.13$ & $0.17 \pm 0.10$ \\
\hline Muscle & $1.60 \pm 1.89$ & $1.34 \pm 0.40$ & $0.49 \pm 0.18$ & $0.45 \pm 0.13$ & $0.24 \pm 0.09$ & $0.06 \pm 0.03$ \\
\hline Bone & $1.09 \pm 0.75$ & $1.03 \pm 0.31$ & $0.51 \pm 0.11$ & $0.39 \pm 0.13$ & $0.21 \pm 0.08$ & $0.07 \pm 0.05$ \\
\hline LNCaP95/PC3 & 0.9 & 3.2 & 3.3 & 2.1 & 1.1 & 1.2 \\
\hline LNCaP95/blood & 0.01 & 3.58 & 3.74 & 4.38 & 3.12 & 2.22 \\
\hline LNCaP95/liver & 0.04 & 0.29 & 0.24 & 0.25 & 0.21 & 0.14 \\
\hline LNCaP95/kidney & 0.03 & 0.71 & 0.96 & 1.00 & 0.85 & 0.92 \\
\hline LNCaP95/muscle & 0.22 & 1.72 & 2.51 & 2.57 & 2.56 & 2.98 \\
\hline \multicolumn{7}{|l|}{ Tumor weight } \\
\hline LNCaP95 (g) & $0.39 \pm 0.29$ & $0.12 \pm 0.11$ & $0.31 \pm 0.17$ & $0.34 \pm 0.13$ & $0.30 \pm 0.15$ & $0.32 \pm 0.19$ \\
\hline РС3 (g) & $0.09 \pm 0.05$ & $0.08 \pm 0.07$ & $0.08 \pm 0.03$ & $0.10 \pm 0.04$ & $0.10 \pm 0.02$ & $0.12 \pm 0.02$ \\
\hline
\end{tabular}

Tumor and normal organ uptakes are expressed as percent of the injected dose per gram (\%ID/g). Values are shown as mean \pm SD. ND, not determined.

and specifically to AR in the LNCaP95 cells (Figure 4D). There was no indication that ${ }^{123}$ I-EPI-002 was a random alkylator, consistent with previous reports that EPI-001 and EPI-002 do not react with glutathione at physiological $\mathrm{pH}(23,29)$. These data support the specificity of ${ }^{123}$ I-EPI-002 binding AR AF-1.

In vivo biodistribution of ${ }^{123}$ I-EPI-002. Biodistribution studies of ${ }^{123}$ I-EPI-002 were conducted in male castrated NOD/SCID mice that each carried both a LNCaP95 and a PC3 xenograft. The LNCaP95 cell line was selected as the target CRPC xenograft due to its high level of expression of endogenous full-length AR and AR-V7. PC3 was selected as the nontarget xenograft because it has very low/negligible protein levels of AR. Table 1 outlines the in vivo tissue biodistribution results of ${ }^{123} \mathrm{I}-\mathrm{EPI}-002$. The cLogP of ${ }^{123} \mathrm{I}-\mathrm{EPI}-002$ is 4.2 , which means it is highly lipophilic, a property that predicts it would be eliminated by the hepatobiliary system. Consistent with this projection, ${ }^{123}$ I-EPI-002 accumulation was highest in the gallbladder, liver, and intestines (Table 1). At 4 hours after injection, 66 percent of the injected dose per gram $(66 \% \mathrm{ID} / \mathrm{g})$ of ${ }^{123}$ I-EPI-002 was detected in the large intestine. Generally, there was low uptake and fast washout from nonspecific tissues.

Accumulation of ${ }^{123}$ I-EPI-002 in LNCaP95 tumors did not support that it was binding irreversibly in this or any other tissues (Table 1). To date, covalent binding of EPI to AR NTD has only been shown in vitro and in closed systems over a long time $(23,24)$. Binding of EPI-002 is proposed as a 2-step process: a quick reversible binding, followed by a very slow covalent binding $(23,24)$. It may be plausible that the short $t_{1 / 2}$ of EPI in vivo ( $\sim 3$ hours) may result in negligible covalent binding because it is not around long enough for covalent binding to occur.

${ }^{123} \mathrm{I}$-EPI-002 reached a maximum $2.2 \% \pm 0.5 \% \mathrm{ID} / \mathrm{g}$ uptake in LNCaP95 tumors at 1 hour after injection. This was greater than the maximum achieved for ${ }^{18} \mathrm{~F}$-FDHT $(0.432 \% \pm 0.183 \% \mathrm{ID} / \mathrm{g} \pm$ SEM) in the target tissue of ventral prostate in rodents at 1 hour after injection (30). Both ${ }^{123}$ I-EPI-002 and ${ }^{18} \mathrm{~F}$-FDHT target the nuclear AR. Based on similar studies with ${ }^{18} \mathrm{~F}$-FDHT, the accumulation of ${ }^{123}$ I-EPI-002 in AR-rich tissues is expected for an AR-mediated process (31). At this time point, ${ }^{123} \mathrm{I}$-EPI-002 uptake in AR-deficient PC3 tumors was only $0.7 \% \pm 0.4 \% \mathrm{ID} / \mathrm{g}$, providing a ratio of 3.2 
for AR-rich tissue (LNCaP95) compared with AR-deficient tissues (PC3). LNCaP95 tumor to blood ratio was $3.58 \pm 0.66$ (Figure $5 \mathrm{~A}$ ), which was similar to the 3.5 ratio achieved with ${ }^{18} \mathrm{~F}$-FDHT, which is used clinically (31). LNCaP95 tumor-blood and tumor-muscle ratios were greater than that of PC3 for all time points examined. Blocking with excess cold EPI-002 (50 mg/kg body weight) caused a $74 \%$ decrease $(P<0.001)$ in ${ }^{123}$ I-EPI-002 accumulation in the LNCaP95 xenograft (Figure 5B), suggesting that the uptake of ${ }^{123}$ I-EPI-002 was AR mediated. This $74 \%$ drop in LNCaP95 tumors by blocking with excess cold EPI-002 was similar to the 70\% drop achieved with testosterone to block ${ }^{18} \mathrm{~F}$-FDHT (31). Blocking with excess cold EPI-002 had no significant effect on levels of ${ }^{123}$ I-EPI-002 uptake in blood, muscle, and PC3 xenograft (Figure 5B). These data suggest that the accumulation of ${ }^{123}$ I-EPI-002 in LNCaP95 xenografts was specifically AR mediated.

Micro-SPECT/CT imaging of ${ }^{123}$ I-EPI-002. On the basis of the results from the biodistribution study and ratios of target tissues to nontarget tissues that were at similar to those achieved with clinically employed ${ }^{18} \mathrm{~F}$-FDHT, a whole-body micro-SPECT/CT study was performed to evaluate the specific tumor-targeting ability of ${ }^{123}$ I-EPI-002. To do this, male castrated NOD/SCID mice bearing both LNCaP95 and PC3 xenografts of approximately equal volumes were scanned using SPECT. Scanning at 2 hours after tail vein injection of ${ }^{123}$ I-EPI-002, when the tumor-blood and tumor-muscle ratios were sufficiently high, provided SPECT images shown in Figure 5C. ${ }^{123}$ I-EPI-002 distinguished LNCaP95 tumors from PC3 tumors, whereas the liver region had relatively higher uptake (Figure 5C; Unblock). The amount of radiopharmaceutical uptake seen in the liver, which was concurrent with the low amount of gastrointestinal uptake, was likely due to the lipophilic nature of ${ }^{123} \mathrm{I}$-EPI-002 and was consistent with the in vivo biodistribution data. LNCaP95 tumor uptake was similar to the tumor uptake observed in the biodistribution study $(1.09 \% \pm 0.41 \% \mathrm{ID} / \mathrm{g}$ at 2 hours after injection, $n=$ 3). In addition, the ${ }^{123}$ I-EPI-002 tracer accumulation was absent from SPECT images when blocked with excess cold EPI-002 (Figure 5C; Block). Importantly, the thyroid uptake was approximately $2.31 \% \pm 0.59 \% \mathrm{ID} / \mathrm{g}$ and was not altered by blocking with EPI-002 $(2.32 \% \pm 0.33 \% \mathrm{ID} / \mathrm{g})$. These data suggest that ${ }^{123} \mathrm{I}-\mathrm{EPI}-002$ may not be metabolically stable and that the ${ }^{123} \mathrm{I}$ may fall off the compound to accumulate in the thyroid.

Histological analysis showed that LNCaP95 xenografts have more necrotic centers than PC3 xenografts (Figure 5D). This suggests that poor vascularization and consequent central necrosis may prevent optimal access of ${ }^{123}$ I-EPI-002 to AR in LNCaP95 tumors. Western blot analysis using an antibody against the AR NTD and V7 confirmed the expression of full-length AR and V7 in LNCaP95 xenografts, which were undetectable in PC3 xenografts (Figure 5E). These data support that ${ }^{123}$ I-EPI-002 accumulation in the LNCaP95 tumor was specific to full-length AR and AR-Vs. Together, all of these data support the feasibility of developing molecular imaging agents directed to AR AF-1 to detect the AR and AR-Vs to improve the clinical management of prostate cancer.

\section{Discussion}

Bone scans are routinely used for metastatic CRPC but they do not provide insight about the cancer itself or whether the lesions are AR positive to aid in treatment selection. Although bone metastates are the predominate site of dissemination, approximately $20 \%-30 \%$ of metastatic CRPC patients have visceral metastases $(32,33)$. If visceral metastases are positive for full-length $A R$, they could benefit from hormone therapies that target AR LBD, such as enzalutamide (34). Even so, approximately one-third of CRPC patients will have intrinsic resistance, and eventually all patients will acquire resistance to enzalutamide or abiraterone (1-4). The major mechanisms of resistance to these therapies include gain-of-function mutations in AR LBD and/or expression of constitutively active AR-Vs that lack LBD (5-7). An important clinical example is drawn from a report using CTCs from CRPC patients, which revealed that, upon initiating enzalutamide treatment, those patients with CTCs that express V7 have a lower PSA response rate, shorter PSA-progression-free survival, shorter clinical or radiographic progression-free survival, and reduced overall survival compared with patients that had CTCs that were V7 negative (5). Compared with taxanes, hormone therapies are the preferred therapeutic due to low toxicity. However, taxanes are more efficacious than AR LBD-targeting therapies in V7-positive CRPC patients (18). Therefore, the ability to distinguish those patients that express AR-Vs that are resistant to AR LBD therapies would be a significant advance in the clinical management of CRPC.

To date, assays to detect AR-Vs are still in early developmental stages, with most focused upon detection of AR-Vs in CTCs. This is because biopsy of the multiple lesions that predominate in the bone of CRPC patients is not viable, making the comprehensive assessment of expression of AR-Vs in metastatic tissues 
A Tumor/blood or Tumor/muscle ratios

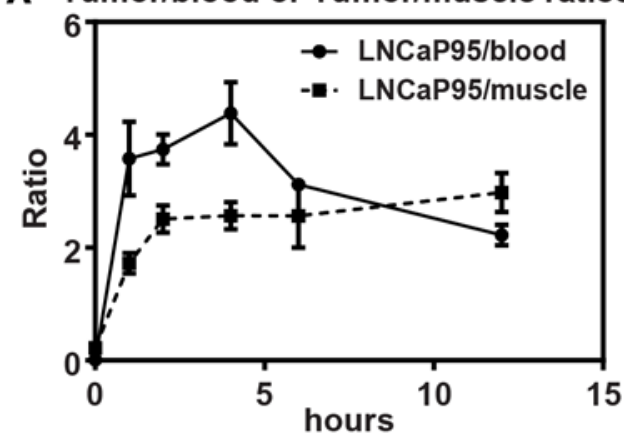

C
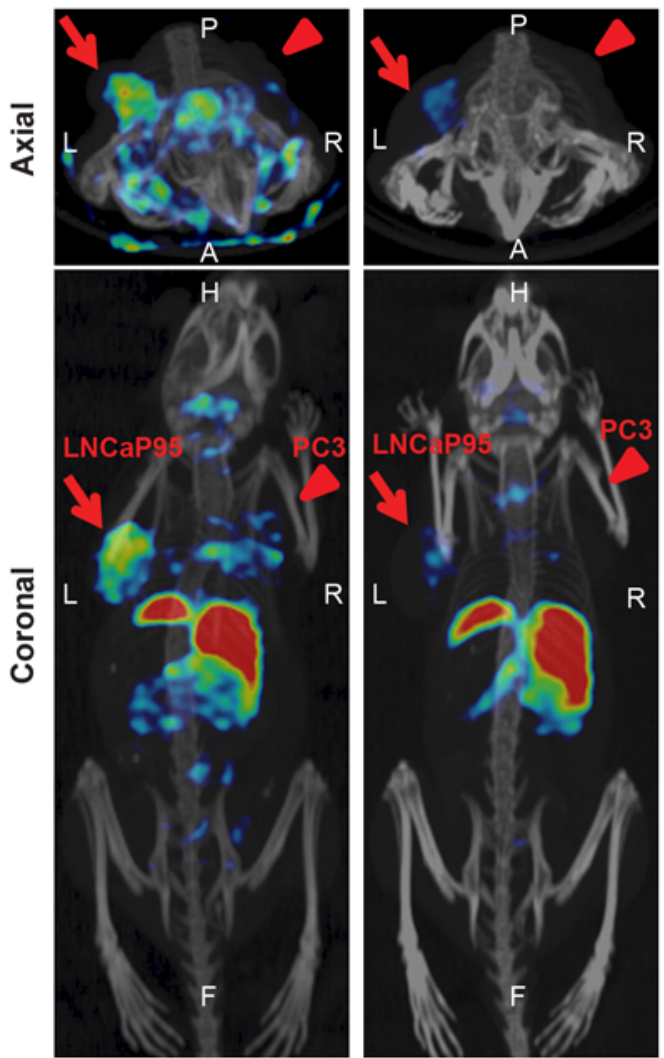

Unblock

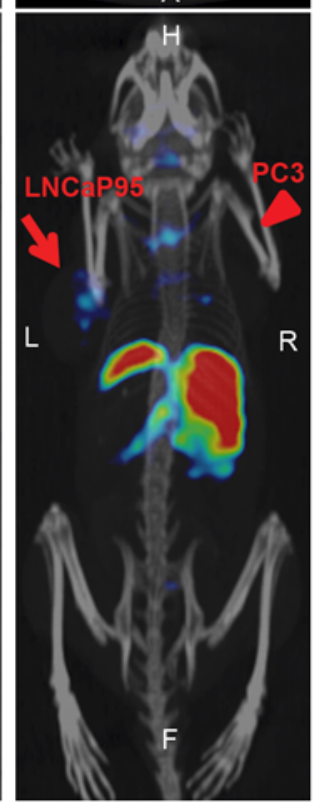

Block
B

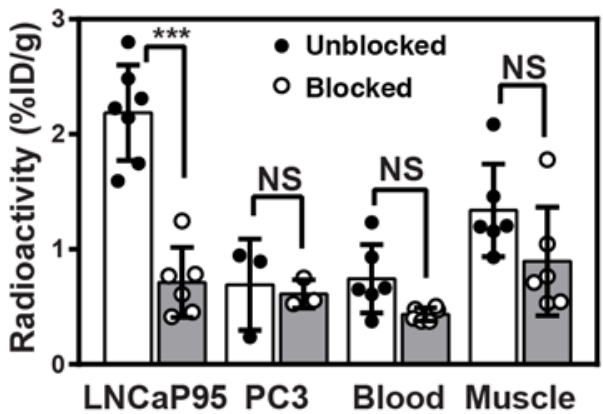

D

High

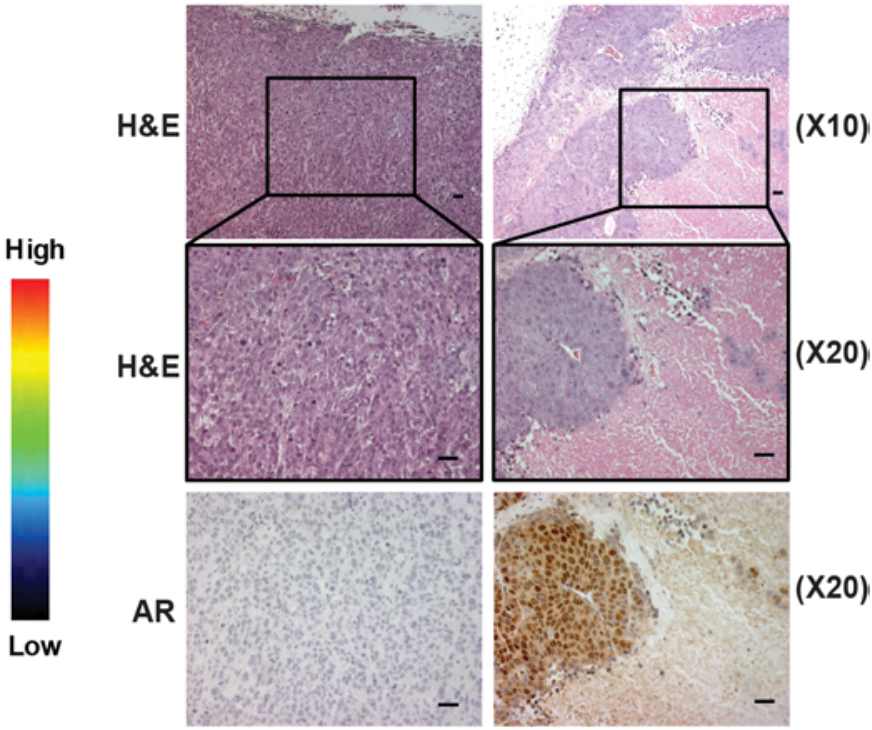

H\&E

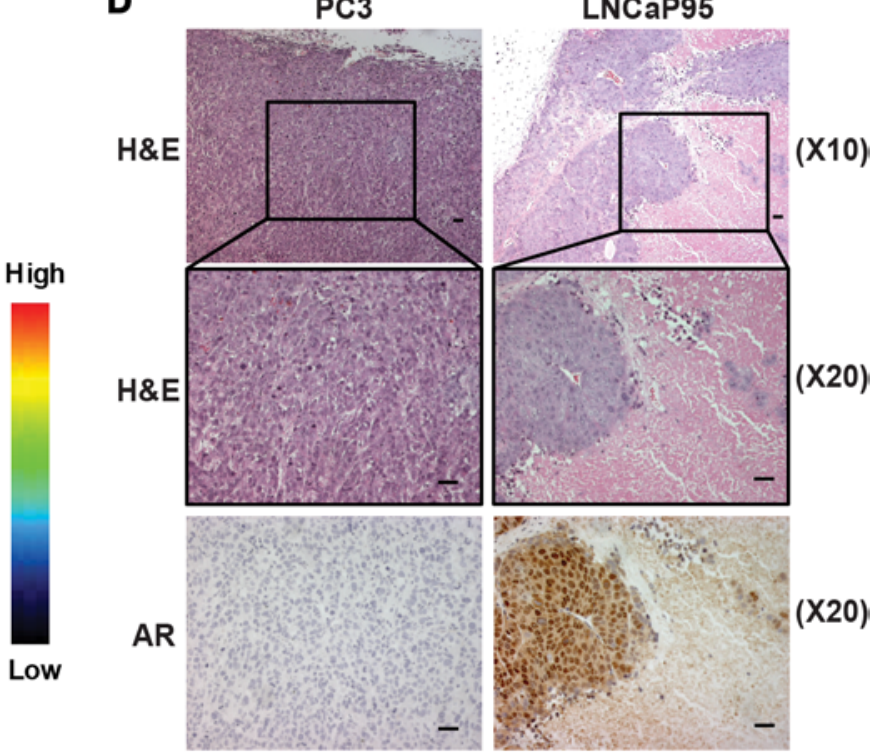

LNCaP95

E

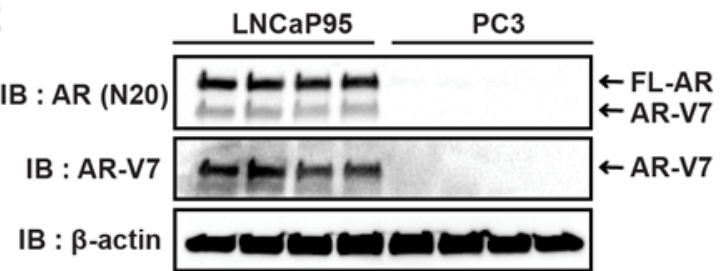

Figure 5. ${ }^{123}$-EPI-002 shows uptake in xenografts that endogenously express ARs. (A) Tumor-blood and tumor-muscle ratios of LNCaP95 xenografts. $n=3-8$ at each time point. Data are shown as mean \pm SEM. (B) Effects of EPI-002 cotreatment on ${ }^{123}$-EPI-002 accumulation in blood, muscle, LNCaP95, and PC3 xenografts (1-hour treatment). The accumulation of ${ }^{123}$-EPI-002 in LNCaP95 xenografts was significantly decreased by blocking with EPI-002. $n=3-7$. Data represent mean $\pm S D$. ${ }^{* *} P<0.001$. One-way ANOVA Dunnett's multiple comparison test was utilized. (C) Axial and coronal micro-SPECT/CT images of castrated NOD/SCID mice bearing LNCaP95 (arrow) and PC3 (arrowhead) xenografts 2 hours after coinjection of ${ }^{123}$ I-EPI-002 with (Block) or without excess dose of EPI-002 (Unblock). The images are scaled to the same threshold. Color scale indicates the level of radioactivity. $n=2$. (D) H\&E staining and immunohistochemistry (AR) of representative sections of formalin-fixed, paraffin-embedded LNCaP95 and PC3 xenografts with lower magnification. Xenografts were extracted approximately 6 weeks after subcutaneous injection of LNCaP95 and PC3 cell lines. The histological analysis demonstrated that LNCaP95 cells that expressed the AR formed a largely concentrated tumor, which resulted in a necrotic center. Scale bar: $40 \mu \mathrm{m}$. (E) Protein expression obtained from LNCaP95 and PC3 xenografts. Four xenografts from each cell line are shown. Western blot analysis of LNCaP95 xenografts showing both full-length AR and AR-V7 expression, both of which were negative in PC3 xenografts. $\beta$-Actin is shown as a protein loading control. Refer to full and uncut gel images in Supplemental Materials.

unfeasible. Clinical application of measurement of expression of AR-Vs using CTCs has several caveats that include abilities to isolate or detect CTCs and the sensitivity of the assay to measure levels of AR-Vs. Thus, a noninvasive imaging approach that could robustly reveal the presence of full-length AR and AR-Vs in all metastatic lesions is of utmost importance to identify patients that may or may not benefit from further antiandrogen or androgen ablation therapies. 
The optimal qualities for a potentially useful imaging probe to provide a noninvasive approach to localize metastatic lesions that express full-length AR and AR-Vs are a high binding affinity to AR NTD and low nonspecific binding affinity. Here, we propose developing an analog of EPI-002 as an AR NTD-targeted molecular imaging probe to detect all AR species. The fact that AR protein is commonly overexpressed in CRPC tissues supports the application of AR NTD-based imaging agents. We show here that I-EPI-002 (a) has 10 times greater potency than EPI-002; (b) maintained specificity for AR; (c) bound to the target, AR AF-1, to yield loss of functional transcriptional activities as expected; and (d) most importantly, that in vivo ${ }^{123} \mathrm{I}$-EPI-002 enabled specific visualization of xenografts that express full-length AR and AR-Vs. I-EPI-002 is potentially the first imaging agent that can directly detect both full-length AR and AR-Vs. Importantly, these data also support the specificity of EPI-002 for AR.

The addition of a substituent in EPI-002 chemical structure could alter the binding of the probe to AR directly or by conformational effects. Here, the addition of iodine to EPI-002 had favorable effects on blocking the transcriptional activity of AR NTD without losing its specificity. The fact that ${ }^{123}$ I-EPI-002 binds the AR NTD and does not affect binding of ligand to AR LBD is an important attribute for an imaging agent for this indication and molecular target. This is because imaging agents are applied at microdoses and hence are highly susceptible to competing agents For most applications, it is predicted that patients failing enzalutamide or other antiandrogens may have an AR LBD occupied by the antiandrogen. Washout of enzalutamide takes approximately 5 half-lives ( $t_{1 / 2}=6-9$ days) or more than 1 month. In addition, if there is steroid hormone breakthrough in castrated or abiraterone-treated patients, or a mutation in AR LBD making the receptor promiscuous to binding other steroid, AR LBD could be occupied by steroid. Under these clinical conditions, an imaging agent that binds to AR NTD would theoretically not be affected, contrary to ${ }^{18} \mathrm{~F}$-FDHT, which competes for binding to LBD and has been shown to have diminished uptake at the tumor site in the presence of testosterone (35). The quality of competing for the AR LBD can, however, be beneficial, as in application of ${ }^{18} \mathrm{~F}$-FDHT as a pharmacodynamic marker to demonstrate the effects of agents targeting the full-length AR (1). Most importantly, in order to distinguish full-length AR from AR-Vs, an imaging agent that binds to the AR NTD, such as ${ }^{123}$ I-EPI-002, which detects full-length AR and AR-Vs, would be required to be used in combination with an imaging agent that only detects full-length $\mathrm{AR}$, such as ${ }^{18} \mathrm{~F}$-FDHT. By using sequential imaging, a discordant distribution or discordant level of uptake between ${ }^{18} \mathrm{~F}-\mathrm{FDHT}$ and a radiolabeled AR NTD imaging agent would indicate the presence of AR-Vs. This experiment was beyond the scope of these proof-of-concept studies; but, in the future, this experiment can be carried out with improved analogs. Such improvements that are currently underway encompass synthesizing a metabolically more stable imaging agent to use in place of ${ }^{123}$ I-EPI-002 and moving away from an iodocompound. However, ${ }^{124}$ I-EPI-002 might be useful for PET imaging and an ${ }^{125}$ I-EPI-002 might be useful as a Auger emitter for therapeutic applications. In the event that an AR NTD-targeted molecular imaging probe has appropriate uptake and biodistribution, it could be further developed as a targeted radiotherapeutic to deliver $\alpha$ - or $\beta$-emitting radionucleotides to lesions that express full-length AR and/or AR-Vs in patients with metastatic CRPC.

In conclusion, these studies suggest that an AR NTD-targeted molecular imaging probe has significant potential for monitoring treatment response; providing insight into the role of all AR species, including AR-Vs, in resistance mechanisms of CRPC; and may provide a means of targeted radiotherapy.

\section{Methods}

Cells, plasmids, transfections, and reporter assays. The synthetic androgen R1881 was purchased from Perkin-Elmer. EPI-002 was provided by NAEJA. Enzalutamide was purchased from Omega Chem. Progesterone (4-pregnene-3,20-dione) was obtained from Steraloids Inc. Dexamethasone and HEPES were obtained from Sigma-Aldrich. LNCaP, PC3, and DU145 cell lines as well as PSA(6.1 kb) luciferase, probasin luciferase, PRE luciferase, GRE luciferase, ERE luciferase, AR-V7 and AR-V567es plasmids, and transfection of cells have been described previously $(22,23,26,29)$. LNCaP cells were obtained from Leland Chung (Cedars-Sinai Medical Center, Los Angeles, California, USA) in September 1993. DU145 cells were from Victor Ling (British Colombia Cancer Agency, Integrative Oncology) in October 1998. LNCaP95, an androgen-independent cell line that expresses full-length AR and AR-V7, was provided by Stephen R. Plymate (University of Washington, Seattle, Washington, USA) in February of 2012 and reported in previous studies (12, 14, 36, 37). PC3 cells were purchased from ATCC. LNCaP95 cells were not authenticated in our laboratory but were regularly tested to ensure that they were mycoplasma free (VenorTMGeM Mycoplasma Detection kit, 
Sigma-Aldrich). LNCaP, PC3, and DU145 cells were authenticated by short tandem repeat analysis and tested to ensure that they were mycoplasma free by DDC Medical in September 2013. All cells used in the experiments were passaged in our laboratory for fewer than 3 months after resurrection.

General procedures for synthesis of I-EPI-002 and $\left.{ }^{123} \mathrm{I}-\mathrm{EPI}-002 .{ }^{123} \mathrm{I}\right] \mathrm{NaI}$ was purchased from Nordion. (S)-1,2-isopropyilideneglycerol, 4-(dimethylamino)pyridine, pyridine, $N, N$-dimethylformamide, $p$-tolunesulfonyl chloride, bisphenol A, sodium iodide, sodium perchlorate, sodium hydroxide, HPLC grade methanol, sodium hydride, $(2 R)$-glycidyl tosylate, cerium chloride heptahydrate, HPLC grade acetonitrile, phosphate buffer, and chloramine-T were purchased from Sigma Aldrich. Purification and quality control of ${ }^{123}$ I-labeled compounds were performed on an Agilent HPLC System equipped with a model 1200 quaternary pump, a model 1200 UV absorbance detector (set at $220 \mathrm{~nm}$ ), and a Bioscan NaI scintillation detector. The radiodetector was connected to a Bioscan B-FC-1000 Flow-count System, and the output from the Bioscan Flow-count system was fed into an Agilent 35900E Interface, which converted the analog signal to digital signal. The operation of the Agilent HPLC system was controlled using the Agilent ChemStation software. The HPLC columns used were a semipreparative column (Phenomenex Luna C18, $5 \mu, 250 \times 10$ $\mathrm{mm}$ ) and an analytical column (Phenomenex Luna C18, $5 \mu, 250 \times 4.6 \mathrm{~mm}$ ). Radioactivity of ${ }^{123}$ I-labeled compounds was measured using a Capintec CRC-25R/W dose calibrator. Details are available in the Supplemental Experimental Procedures.

Fluorescence polarization. Androgen, PR, ER, and GR PolarScreen Competitor Assay kits (Invitrogen) were used according to the manufacturer's protocol. Serial dilution was done for each small molecule, and solvent was compensated to ensure equal volume of DMSO and ethanol in each sample. Fluorescence polarization at excitation wavelength $470 \mathrm{~nm}$ and emission at $530 \mathrm{~nm}$ was measured in Greiner 384 black clear-bottomed plates using Infinite M1000 (TECAN).

Viability and proliferation assays. PC 3 cells (2,000 cells/well), DU145 cells (2,000 cells/well), and LNCaP cells $(5,000$ cells/well) were plated in 96 -well plates in respective media plus $0.5 \%$ FBS. The next day, PC3 and DU145 cells were treated with DMSO (vehicle control) and I-EPI-002 for 3 days, and LNCaP cells were pretreated with vehicle and I-EPI-002 for 1 hour before treatment with $0.1 \mathrm{nM}$ R1881 for 4 days. Proliferation and cell viability was measured using alamarBlue Cell Viability Assay (Invitrogen) following the manufacturer's protocol.

For BrdU incorporation experiments, LNCaP95 cells (8,000 cells/well) were seeded in 96-well plates for 48 hours in RPMI with 10\% charcoal stripped serum, which was changed to serum-free media 24 hours before treatment with $5 \mu \mathrm{M}$ enzalutamide, $25 \mu \mathrm{M}$ EPI-002, $2 \mu \mathrm{M}$ I-EPI-002, or serial concentrations of I-EPI-002. BrdU incorporation was measured after 2 days using the BrdU ELISA kit (Roche Diagnostics) according to the manufacturer's protocol.

FACS analysis. LNCaP95 cells (75,000 cells/dish) were plated in 10-cm dishes and were treated with inhibitors under serum-free and phenol red-free conditions for 48 hours. Cells were pulse labeled with 10 $\mu \mathrm{M}$ BrdU for 2 hours and fixed in 70\% ethanol. BrdU-labeled cells were probed with anti-BrdU-FITC antibody (BD Biosciences), and DNA was stained with 7-aminoactinomycin D (Sigma-Aldrich). Data were acquired using a FACS Calibur (BD Biosciences). Bivariate analysis was performed using FlowJo 7 software (Ashland).

Binding assays. The cell-free binding assay was performed with commercially available full-length AR recombinant protein or AR AF-1 recombinant protein that was expressed and purified as previously described $(22,38)$, with additional purification by size exclusion chromatography. Binding reactions between EPI-002 or DMSO (vehicle control) and the recombinant AR AF-1 (amino acid residues 142-485) or fulllength AR were carried out by mixing $60 \mu \mathrm{M}$ EPI-002 or DMSO with $10 \mu \mathrm{M}$ AR AF-1 and $1 \mu \mathrm{M}$ EPI-002 or DMSO with $0.35 \mu \mathrm{M}$ full-length $\mathrm{AR}$ in equivalent amounts. Binding reaction was incubated at room temperature for 6 hours. $200 \mu \mathrm{Ci}$ of ${ }^{123}$ I-EPI-002 was then mixed in each sample at room temperature for 16 hours prior to SDS-PAGE. Proteins were separated from free probes on $8 \%$ SDS-PAGE, and radioactivity was visualized using Fujifilm FLA-7000 image analyzer (GE Healthcare). The same gel was stained with Coomassie blue R-250. To examine binding to the endogenous AR, LNCaP95 cells (60,000 cells/well) were seeded in 12-well plates and serum starved for 24 hours before treating cells for 16 hours with DMSO or 25 $\mu \mathrm{M}$ EPI-002 and with $400 \mu \mathrm{Ci}$ of ${ }^{123}$ I-EPI-002. Proteins were extracted from treated cells with RIPA buffer containing $50 \mathrm{mM}$ tris ( $\mathrm{pH} 8.0$ ), $150 \mathrm{mM} \mathrm{NaCl}, 0.5 \% \mathrm{Na}$ Deoxycholate, 1\% NP-40, 0.1\% SDS, and EDTAfree protease inhibitors. ${ }^{123}$ I-EPI-proteins were separated by SDS-PAGE, and radioactivity was visualized using Fujifilm FLA-7000 image analyzer, and subjected to Western blot analysis using anti-AR antibody. 
Immunohistochemistry and Western blot analysis. Immunohistochemistry was performed as previously described $(22,23)$. For analysis of AR protein, concentrations of lysates of homogenized LNCaP95 and PC3 xenografts were measured by bicinchoninic acid assay after albumin depletion. Proteins (20 $\mu \mathrm{g})$ were resolved on a NuPAGE $4 \%-15 \%$ Bis Tris gradient gel, transferred to nitrocellulose membrane, and probed for AR species using antibodies to the AR NTD and AR-V7. LNCaP95 cells (250,000 cells/well) were seeded in a 6-well plate for 48 hours and serum-starved for 24 hours, followed by treatment with DMSO, enzalutamide $(5 \mu \mathrm{M})$, EPI-002 $(25 \mu \mathrm{M})$, or I-EPI-002 $(5 \mu \mathrm{M})$ for 48 hours. Cells were harvested, and wholecell lysate (10 to $15 \mu \mathrm{g}$ ) was subjected to SDS-PAGE. Antibodies used were as follows: AR N-20 (sc-816, Santa Cruz Biotech Inc.); AR-V7 (AG10008, Precision Antibody); UBE2C (A-650, BostonBiochem); and cyclinD1, cyclinD3, p27kip1, CDK2, CDK4, and CDK6 from the Cell Cycle Regulation Sampler Kit (9932 from Cell Signaling Technology). $\beta$-Actin (ab6276, Abcam) was used as a loading control.

Xenografts and animal biodistribution studies. Six- to eight-week-old male NOD/SCID mice were castrated 2 weeks before inoculating LNCaP95 cells (10 million cells/tumor) and PC 3 cells ( 2 million cells/ tumor) subcutaneously. The mice bearing LNCaP95 and PC3 xenografts were injected with $370 \mathrm{kBq}$ to 7.4 MBq (10-200 $\mu \mathrm{Ci})$ of ${ }^{123}$ I-EPI-002 in $200 \mu \mathrm{l}$ of $30 \%$ PEG solution via the tail vein. At $0,1,2,4,6$, and 12 hours after injection, blood, liver, kidney, spleen, lung, large intestine, small intestine, stomach, heart, muscle, bone, bladder, brain, gallbladder, and tumor samples were collected and weighed and their radioactivity content was determined using an automated $\gamma$-spectrometer. The results were expressed as \% $\mathrm{ID} / \mathrm{g}$. More than 3 animals per time point were used.

Micro-SPECT/CT imaging. SPECT/CT studies were performed using the MILabs U-SPECT- II (Advanced Molecular Vision). LNCaP95 and PC-3 tumor-bearing mice (20-25 g) were anesthetized with isoflurane inhalation at $2 \%$ and placed on a heating pad to maintain body temperature. The mice were then injected with ${ }^{123}$ I-EPI-002 (37-74 MBq; $1-2 \mathrm{mCi}$, in $200 \mu \mathrm{l}$ of $30 \%$ PEG solution) via the tail vein. A competition experiment was performed by coinjection of the radiotracer with $50 \mathrm{mg} / \mathrm{kg}$ of EPI-002 in a total volume of $200 \mu \mathrm{l}$ of $30 \%$ PEG solution. SPECT scans were acquired over 30 minutes and 2 frames at 15 minutes each using 1.0-mm multi-pinhole collimator, after which CT scans were performed for anatomic reference (parameters; $60 \mathrm{kV}, 600 \mu \mathrm{A}$ ). SPECT imaging data were reconstructed using MI Labs reconstruction software ( 2 subsets, 30 iterations). PMOD software was used to analyze and view the images, and a Gaussian filter was applied after reconstruction.

Statistics. Statistical analysis was performed using GraphPad Prism (version 6.01 GraphPad Software Inc.). Values are presented as mean $\pm \mathrm{SD}$ or mean \pm SEM. Except where specified, comparisons between groups were performed with 1-way ANOVA Dunnett's multiple comparisons test, and differences were consider statistically significant at $P$ values less than 0.05 .

Study approval. All experiments involving animals conformed to the relevant regulatory and ethical standards established by the University of British Columbia Animal Care Committee. The University of British Columbia Animal Care Committee approved the experiments.

\section{Author contributions}

YI acquired and analyzed the data and wrote the manuscript. AHT acquired and analyzed the data. JP synthesized the compound. JKL performed BrdU assay. CAB performed FACS analysis. KJ administrated technical or material support. JW performed animal studies. NRM analyzed the data and administrated material support. JGF administrated technical or material support. K-SL supervised and administrated material support. RJA developed the chemical compound. MDS conceived of the concept; developed, designed, and supervised the work; and wrote the manuscript. All the authors approved the paper.

\section{Acknowledgments}

We thank Francois Benard for technical advice and Navjit Hundal-Jabal for technical assistance. This research was supported by a grant to MDS from US National Cancer Institute (2R01CA105304).

Address correspondence to: Marianne D. Sadar, Department of Genome Sciences Centre, British Columbia Cancer Agency, 675 West 10th Avenue, Vancouver, British Columbia, Canada V5Z1L3. Phone: 604.675.8157; E-mail: msadar@bcgsc.ca. 
1. Scher HI, et al. Antitumour activity of MDV3100 in castration-resistant prostate cancer: a phase 1-2 study. Lancet. 2010;375(9724):1437-1446.

2. Scher HI, et al. Increased survival with enzalutamide in prostate cancer after chemotherapy. N Engl J Med. 2012;367(13):11871197.

3. de Bono JS, et al. Abiraterone and increased survival in metastatic prostate cancer. N Engl J Med. 2011;364(21):1995-2005.

4. Ryan CJ, et al. Abiraterone in metastatic prostate cancer without previous chemotherapy. N Engl J Med. 2013;368(2):138-148.

5. Antonarakis ES, et al. AR-V7 and resistance to enzalutamide and abiraterone in prostate cancer. N Engl J Med. 2014;371(11):1028-1038.

6. Nadiminty N, et al. NF-kappaB2/p52 induces resistance to enzalutamide in prostate cancer: role of androgen receptor and its variants. Mol Cancer Ther. 2013;12(8):1629-1637.

7. Mostaghel EA, et al. Resistance to CYP17A1 inhibition with abiraterone in castration-resistant prostate cancer: induction of steroidogenesis and androgen receptor splice variants. Clin Cancer Res. 2011;17(18):5913-5925.

8. Jenster G, van der Korput HA, van Vroonhoven C, van der Kwast TH, Trapman J, Brinkmann AO. Domains of the human androgen receptor involved in steroid binding, transcriptional activation, and subcellular localization. Mol Endocrinol. 1991;5(10):1396-1404.

9. Rundlett SE, Wu XP, Miesfeld RL. Functional characterizations of the androgen receptor confirm that the molecular basis of androgen action is transcriptional regulation. Mol Endocrinol. 1990;4(5):708-714.

10. Simental JA, Sar M, Lane MV, French FS, Wilson EM. Transcriptional activation and nuclear targeting signals of the human androgen receptor. J Biol Chem. 1991;266(1):510-518.

11. Guo Z, et al. A novel androgen receptor splice variant is up-regulated during prostate cancer progression and promotes androgen depletion-resistant growth. Cancer Res. 2009;69(6):2305-2313

12. $\mathrm{Hu} \mathrm{R}$, et al. Distinct transcriptional programs mediated by the ligand-dependent full-length androgen receptor and its splice variants in castration-resistant prostate cancer. Cancer Res. 2012;72(14):3457-3462.

13. Li Y, Chan CS, Brand LJ, Hwang TH, Silverstein KA, Dehm SM. Androgen receptor splice variants mediate enzalutamide resistance in castration-resistant prostate cancer cell lines. Cancer Res. 2013;73(2):483-489.

14. Hu R, et al. Ligand-independent androgen receptor variants derived from splicing of cryptic exons signify hormone-refractory prostate cancer. Cancer Res. 2009;69(1):16-22.

15. Dehm SM, Schmidt LJ, Heemers HV, Vessella RL, Tindall DJ. Splicing of a novel androgen receptor exon generates a constitutively active androgen receptor that mediates prostate cancer therapy resistance. Cancer Res. 2008;68(13):5469-5477.

16. Hörnberg E, et al. Expression of androgen receptor splice variants in prostate cancer bone metastases is associated with castration-resistance and short survival. PLOS ONE. 2011;6(4):e19059.

17. Haile S, Sadar MD. Androgen receptor and its splice variants in prostate cancer. Cell Mol Life Sci. 2011;68(24):3971-3981.

18. Antonarakis ES, et al. Androgen Receptor Splice Variant 7 and Efficacy of Taxane Chemotherapy in Patients With Metastatic Castration-Resistant Prostate Cancer. JAMA Oncol. 2015;1(5):582-591.

19. Dawson SJ, et al. Analysis of circulating tumor DNA to monitor metastatic breast cancer. N Engl J Med. 2013;368(13):1199-1209

20. Haber DA, Velculescu VE. Blood-based analyses of cancer: circulating tumor cells and circulating tumor DNA. Cancer Discov. 2014;4(6):650-661.

21. Hu DG, et al. Identification of androgen receptor splice variant transcripts in breast cancer cell lines and human tissues. Horm Cancer. 2014;5(2):61-71.

22. Andersen RJ, et al. Regression of castrate-recurrent prostate cancer by a small-molecule inhibitor of the amino-terminus domain of the androgen receptor. Cancer Cell. 2010;17(6):535-546.

23. Myung JK, et al. An androgen receptor $\mathrm{N}$-terminal domain antagonist for treating prostate cancer. J Clin Invest. 2013;123(7):2948-2960.

24. De Mol E, et al. EPI-001, a compound active against castration-resistant prostate cancer, targets transactivation unit 5 of the androgen receptor [published online ahead of print June 29, 2016]. ACS Chem Biol. doi:10.1021/acschembio.6b00182.

25. Tran C, et al. Development of a second-generation antiandrogen for treatment of advanced prostate cancer. Science. 2009;324(5928):787-790.

26. Sun $\mathrm{S}$, et al. Castration resistance in human prostate cancer is conferred by a frequently occurring androgen receptor splice variant. J Clin Invest. 2010;120(8):2715-2730.

27. Zhang $\mathrm{X}$, et al. Androgen receptor variants occur frequently in castration resistant prostate cancer metastases. PLoS ONE. 2011;6(11):e27970.

28. Yang YC, et al. Targeting androgen receptor activation function-1 with EPI to overcome resistance mechanisms in castration-resistant prostate cancer [published online ahead of print May 2, 2016]. Clin Cancer Res. doi:10.1158/1078-0432.CCR-15-2901.

29. Banuelos CA, et al. Characterization of niphatenones that inhibit androgen receptor $\mathrm{N}$-terminal domain. PLoS ONE. 2014;9(9):e107991.

30. Liu A, Dence CS, Welch MJ, Katzenellenbogen JA. Fluorine-18-labeled androgens: radiochemical synthesis and tissue distribution studies on six fluorine-substituted androgens, potential imaging agents for prostatic cancer. J Nucl Med. 1992;33(5):724-734

31. Bonasera TA, et al. Preclinical evaluation of fluorine-18-labeled androgen receptor ligands in baboons. J Nucl Med. 1996;37(6):1009-1015

32. Pond GR, Sonpavde G, de Wit R, Eisenberger MA, Tannock IF, Armstrong AJ. The prognostic importance of metastatic site in men with metastatic castration-resistant prostate cancer. Eur Urol. 2014;65(1):3-6.

33. Goodman OB Jr, et al. Exploratory analysis of the visceral disease subgroup in a phase III study of abiraterone acetate in metastatic castration-resistant prostate cancer. Prostate Cancer Prostatic Dis. 2014;17(1):34-39.

34. Evans CP, et al. The PREVAIL Study: Primary Outcomes by Site Extent of Baseline Disease for Enzalutamide-treated Men with Chemotherapy-naive Metastatic Castration-resistant Prostate Cancer [published online ahead of print March 19, 2016] Eur Urol. 2016;S0302-2838(16):00274-00278. doi:10.1016/j.eururo.2016.03.017.

35. Larson SM, et al. Tumor localization of 16beta-18F-fluoro-5alpha-dihydrotestosterone versus 18F-FDG in patients with progressive, metastatic prostate cancer. J Nucl Med. 2004;45(3):366-373. 
36. Liu LL, Xie N, Sun S, Plymate S, Mostaghel E, Dong X. Mechanisms of the androgen receptor splicing in prostate cancer cells. Oncogene. 2014;33(24):3140-3150.

37. Yang YC, et al. Spongian diterpenoids inhibit androgen receptor activity. Mol Cancer Ther. 2013;12(5):621-631.

38. Reid J, Kelly SM, Watt K, Price NC, McEwan IJ. Conformational analysis of the androgen receptor amino-terminal domain involved in transactivation. Influence of structure-stabilizing solutes and protein-protein interactions. $J$ Biol Chem. 2002;277(22):20079-20086. 